\title{
Enhancement of medical images using fuzzy logic
}

\author{
Yousra Ahmed Fadil, Baidaa Al-Bander, Hussein Y. Radhi
}

University of Diyala, Ba'qubah, Iraq

\begin{tabular}{l} 
Article Info \\
\hline Article history: \\
Received May 24, 2021 \\
Revised Jul 29, 2021 \\
Accepted Aug 7, 2021 \\
\hline
\end{tabular}

\section{Keywords:}

Artificial intelligence

C-means clustering

Fuzzy logic

Image enhancement

Membership function

\begin{abstract}
Image enhancement is one of the most critical subjects in computer vision and image processing fields. It can be considered as means to enrich the perception of images for human viewers. All kinds of images typically suffer from different problems such as weak contrast and noise. The primary purpose of image enhancement is to change an image's visual appearance. Many algorithms have recently been proposed for enhancing medical images. Image enhancement is still deemed a challenging task. In this paper, the fuzzy cmeans clustering (FCM) technique is utilized to enhance the medical images. The method of enhancement consists of two stages. The proposed algorithm conducts a cluster test on the image pixels. It then increases the difference of gray level between the diverse objects to accomplish the enhancement purpose of the medical images. The experimental results have been tested using various images. The algorithm enhanced the small target of the image to a reasonable limit and revealed favorable performance. The results of image enhancement techniques were evaluated by using terms of different criteria such as peak signal to noise ratio (PSNR), mean square error (MSE) and average information contents (AIC), showing promising performance.
\end{abstract}

This is an open access article under the CC BY-SA license.

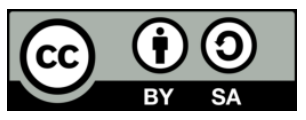

Corresponding Author:

Yousra Ahmed Fadil

University of Diyala, Ba'qubah, Iraq

Email: yousraah2012@gmail.com

\section{INTRODUCTION}

Image enhancement is an algorithm where the quality of degraded images is enhanced and made more precise with the help of different image enhancement algorithm. The fundamental goal of image enhancement is to improve human vision by improving the observation of features in the image. The enhancement processing helps to maximize the purity sharpness and specifics of features of interest towards information extraction and further analysis [1]. Due to the importance of the medical imaging field in different areas of life, especially in cases of illnesses experienced by the human, it is essential to find methods, techniques, and algorithms to process these images and reduce the noise and other details that make the image distorted and unclear [2], [3]. Medical images became very important for human life. These images should be carefully taken as much as possible by doctors because it affects the disease diagnosis procedure and physician's decisions [4]. Several processing algorithms have been introduced, including image enhancement algorithms, making image properties more visible and clearer to the human eye. Examples of this are high contrast, image edge enhancement, and noise elimination.

Some of the techniques and algorithms include non-linear pyramidal, multi-scale morphological, retinex theory, mountain clustering, or how contrast can be improved by segmentation and morphological operators, K-sigma clipping, curvelet transformations, fuzzy logic, wavelet transformations, and genetic algorithms [5]. The fuzzy logic represents one of the most important techniques used to improve images, which has been used extensively in various applications. It has spread significantly and increasingly in several areas, especially the field of improvement and processing of images. The main reason is the lack of 
complexity in the mathematical relationships used in its construction, words in calculations instead of numbers and controls sentences instead of mathematical equations [6]. It is well known that humans cannot deal with large amounts of numerical information compared with a computer which has a high possibility of solving various complex calculations in short times. However, the computer is incapable of solving issues that cannot be represented numerically. At the same time, human can solve these problems, which make scientists search for techniques that simulate the human intelligence system with the tremendous development in computers and software science. In our practical life, we often encounter phenomena in which we use concepts with more than one possibility that can be solved using a fuzzy logic system [7].

According to the literature, in [8], multiple forms of histogram equalization approaches are used and evaluated, demonstrating that dynamic histogram equalization (DHE) is a multi-histogram equalization method that is preferred to equalization of bihistogram. Contrast limited adaptive histogram equalization (CLAHE) is a sector histogram equalization process that is superior to traditional AHE. To improve matching performance, extraction, and image enhancement techniques [9] are used to low-resolution images collected with an Optris PI 450 lightweight thermal camera with a 382 × 288 optical resolution performance. Furthermore, in [10], the modified histogram equalization (MHE) technique has been proposed for medical image contrast enhancement, which used wavelet transform and histogram mapping functions to enhance the image visualization, which gave the best contrast enhancement. In [11], the Framete Transform [FT] technique is applied for resolution improvement to the satellite images; FT decomposed input images while inverse FT produces a new resolution enhanced image. Badgujar and Singh in [12], an effective methodological approach for underwater pictures enhancement using the generalized histogram adjustment, discrete wavelet transform is proposed.

AlSaif and Abdullah authors in [13] propose a parametric contrast scale augmentation approach using transform techniques such as the Laplacian pyramid transformation method, discrete wavelet technique, stationary wavelet transformations, and dual-tree complex wavelet transform. Fuzzy technique with triangular membership is used for enhancing the contrast of variety variance and different sizes images, and the results show that the contradiction is increased in [14]. Another work [15], fuzzy logic enchantment and low contrast enhancement color images and contrast enhancement technique using homomorphic refinement with the fuzzy logic concept were presented. Gupta et al. in [16], a fuzzy grayscale enhancement method for low contrast image is presented and generated best quality improved image and used minimal processing resources than other processing methods [17]. Raj et al. in [18] new technique to histogram equalization by modifying the function of probability density. This process gives a goode level of detail in images as a result to the enhancement technique. Moreover, in [19], a new technique is proposed to enhance a color image using contourlet transform and saturation components. In addition, in [20], the algorithm of a CA-based method has demonstrated the method used to rebuild the surface of the liver from low contrast CT pictures.

Despite the extensive research work presented in the literature, the image enhancement issue is still open for further improvements. In this work, the fuzzy c-means clustering (FCM) algorithm is utilized to enhance the medical images. This algorithm increases the difference of gray level between the diverse objects to achieve the enhancement purpose of the images. This has been achieved in two stages depending on managing the cluster test on the image pixels and raising the gray level difference.

\section{RESEARCH METHOD}

\subsection{Histogram equalization}

It is a scheme used to improve contrast enhancement in different applications because of its simple function and efficiency. The benefit of histogram equalization is distributing the gray levels within an image so that each grey is similarly to occur [21]. Histogram equalization will excel the brightness and contrast of dark and faint images. Histogram equalization uses the cumulative density of images to convey the grayness levels of the input image to level of the enhanced one. It works on the fact that the grey level histograms show the bright or the dark peaks. The image brightness can be described as a fuzzy set, including the group dark, bright and gray intensity levels. According to the histogram normalization, the membership degree can be specified. Histogram of the image can be represented as a discrete function [22]. The shape of the histogram gives information about the nature of the image. The histogram of the input image is as steady as possible after applying the histogram equalization method. The histogram equalization technique specifies the number of pixels to each gray level value, where pervasion histogram lets the light pixels seem lighter, and the dark pixels seem darker [23]. The cumulative histogram $\mathrm{c}(\mathrm{x})$ for the histogram $\mathrm{h}(\mathrm{x})$ is defined as:

$$
c(x)=\int_{-\infty}^{x} h(y)
$$




\subsection{Image enhancement by using fuzzy}

Vision applications rely mainly on images enhancement algorithms. Large numbers of techniques have been presented for enhancing the images. In image enhancement, the objective is to emerge a specific image features for subsequent analysis or image use in other applications. Most images typically suffer from imperfections such as poor contrast and low-resolution contrast, and imaging artifacts. Enhancement mechanisms for images such as global stretching and histogram equalization do not all the time give good results, particularly for images with significant variance [24]. According to the importance of the image enhancement principles, many approaches have been yielded in the literature. Most of these methods used for image enhancement are used in a particular domain like histogram equalization. Many methods are used to improve digital images because some images suffer from ambiguity grayness when processed. The fuzzy logic is considered an appropriate technique in dealing with these issues. The principle of fuzzy system deals with approximate values rather than fixed and exact reasoning, and it has been expanded to treat with the notion of uncertainty and partial truth. In general, the fuzzy logic contains three basic steps, begins from the image fuzzification. At this step, image elements that are fragile values are converted to a set value using membership functions where each element is given a degree (membership between 0,1) [25]. The next step is membership modification, where after obtaining the degree of membership, it is updated with a value that fits the type of treatment to be applied to the image. In the final step, image defuzzification, the values are converted to fragile values, and after this step, the results of the required treatment are performed on the image. The image can be represented in a fuzzy matrix as:

$$
F=\bigcup_{i=1}^{M} \bigcup_{j=1}^{N} \frac{P i j}{X i j} \quad \text { with } P \subseteq[0,1]
$$

In the aforementioned equation, $\mathrm{X}_{\mathrm{ij}}$ represents the intensity of the pixel (i, j), pij is the membership degree of the pixel $(i, j)$ where $p_{i j} \in[0,1]$, all $p_{i j}$ consist of the image fuzzy feature plane, $p_{i j}$ can be computed by a fuzzy membership function, different membership functions can produce various effects. Fuzzy image operation may be categorized into three stages; the first step represents the image coding (fuzzification) by a change to an actual scalar value of the image into a fuzzy value. This is carried out with diverse types of membership functions. The second step can be performed by the operation on the membership plane, and the final step can be represented by the defuzzification. In general, the fuzzy plane confined between from 0 and 1 , while the actual gray level intensities range 0 and 255. The 0 value represents the darkest pixel, and the value one represents the brightest pixel. Image threshold has played an important role in image improvement. A fuzzy c-means clustering procedure for image threshold is used for image enhancement. It's a clustering approach that permits one piece of visual data to be linked to many clusters [26].

\subsection{Methodology}

Membership is assigned to each data set according to the center of each cluster, depending on the distance between the cluster center and the data set. After each iteration, the cluster center and the membership are modified. When applying clustering to the data set, crisp classification may be obtained when the clusters are well separated [27]. But in general situation, clusters are not well separated. The fuzzy c-mean clustering can be performed by selecting an initial fuzzy partition, computing the center to each cluster then modifying the fuzzy partition until the center value does not change. The set of $n$ objects $Y=\left\{y_{1}\right.$, $\left.\mathrm{y}_{2} \ldots, \mathrm{y}_{\mathrm{n}}\right\}$, where $\mathrm{y}_{\mathrm{i}}$ is a dimensional data point. A fuzzy clustering $\mathrm{C}_{1}, \mathrm{C}_{2} \ldots, \mathrm{C}_{\mathrm{k}}$ as k-cluster. Each element in the matrix partition $\mathrm{W}_{\mathrm{i}, \mathrm{j}}$ is a weight that describes the degree of membership of an object in cluster $\mathrm{C}_{\mathrm{i}}$ where the matrix $\mathrm{W}=\mathrm{w}_{\mathrm{i}, \mathrm{j}} \in[0,1]$, for $\mathrm{i}=1 \ldots \mathrm{n}$ and $\mathrm{j}=1 \ldots \mathrm{k}$. The main objective of the fuzzy c-means algorithm is to minimize a sum of squared error (SSE); the objective equation can be expressed is being as:

$$
S S E=\sum_{j=1}^{k} \sum_{i=1}^{n} W_{i, j}^{p} \operatorname{dist}\left(X_{i}, C_{i}\right)^{2}
$$

Where $W$ represents the membership matrix, the set of fuzzy clusters acted by the membership matrix $W$, the linked set of cluster center is represented by $C$, and the dist describes the distance between the data $\mathrm{X}$ and center $C$. The fuzzy center is chosen to minimize the variance of the black and white pixels. The membership $W_{i j}$ and the cluster centers $C_{i}$ can be updated by:

$$
W_{i j}=\frac{1}{\sum_{k=1}^{c}\left(\frac{\left\|x_{j}-c_{i}\right\|}{\left\|X_{j}-c_{k}\right\|}\right)^{2 /(m-1)}}
$$




$$
C_{i}=\frac{\sum_{i=1}^{m} U_{i j}^{m} X_{i}}{\sum_{i=1}^{N} U_{i j}^{m}}
$$

Where $m$ represents the fuzzification scale which is a weighting exponent on each membership of fuzzy set. The membership degree in each cluster $(i)$ represented by $\left(\mathrm{x}_{i}\right), x_{i}$ is the dimensional measured data. $d\left(X_{k}, C i\right)$ is a distance between object cluster center $C i$ and the $X_{k},(\|*\|)$ represents a norm of the similarity between the center and any measured data. The fuzzy logic is not only used to maintain the image brightness but also enhance the contrast of the main image. The fuzzy logic could be used to create the fuzzy histogram aiming to improve the inaccuracy of gray level values, and it is separated into clusters. Then each histogram is allocating to a new area. The image fuzzification converts the intensities of the gray level into the fuzzy plane. The value of this plane is lying between 0 and 1

$$
X=\left[\begin{array}{ccc}
\frac{P_{11}}{X_{11}} & \cdots & \frac{P_{1 n}}{X_{1 n}} \\
\vdots & & \vdots \\
\frac{P_{x 1}}{X_{m 1}} & \cdots & \frac{P_{m n}}{X_{m n}}
\end{array}\right]
$$

The $p_{i j}=0$ indicates the dark, and $p_{i j}=1$ indicates bright. The fuzzy c-mean executes the clustering by searching in iterative checking for sets of fuzzy clusters.

\section{RESULTS AND DISCUSSION}

This section displays the comparison between the suggested process and several other processes such as fuzzy logic-based histogram equalization and histogram equalization in terms of objectives and subjective measurements. Figure 1 describes the performance of the suggested approach comparing to the existing methods subjectively. Furthermore, the computational models are used compute the image quality regularly with individual assessments. The image quality assessment metrics applied in this paper have been executed using peak signal to noise ratio (PSNR), mean square error (MSE), entropy or average information contents (AIC). The quality parameter peak signal to noise ratio is clarified as the ratio of the signal's top power to the top power of the noise. PSNR value computation is defined is being as.

$$
\begin{aligned}
& P S N R=10 \log _{10}\left(\frac{M A X_{I}^{2}}{M S E}\right) \\
& =20 \log _{10}\left(\frac{M A X_{I}}{\sqrt{M S E}}\right) \\
& 20 \log _{10}\left(M A X_{I}\right)-10 \log _{10}(M S E) \\
& M S E=\frac{1}{m n} \sum_{i=0}^{m-1} \sum_{j=0}^{n-1}[(i, j)-k(i, j)]^{2}
\end{aligned}
$$

The entropy information contents (AIC) is explained as

$$
A I C=-\sum_{k=0}^{L-1} P(k) \log P(k)
$$

The probability intensity function of the $k$ th gray level was defined by $P(k)$. Figure $1(a)$ represents the original grayscale image. Figure 1(b) represents the resulted images obtained by the proposed method. Figure 1(c) represent the images produced from the fcnBPDFHE method. The results that have been computed using the image quality assessment metrics applied in this paper show that the proposed method has improved the images depend on the results found in Table 1. Moreover, Figure 2 explores the simulation results of the proposed methods comparing to the existing methods. 

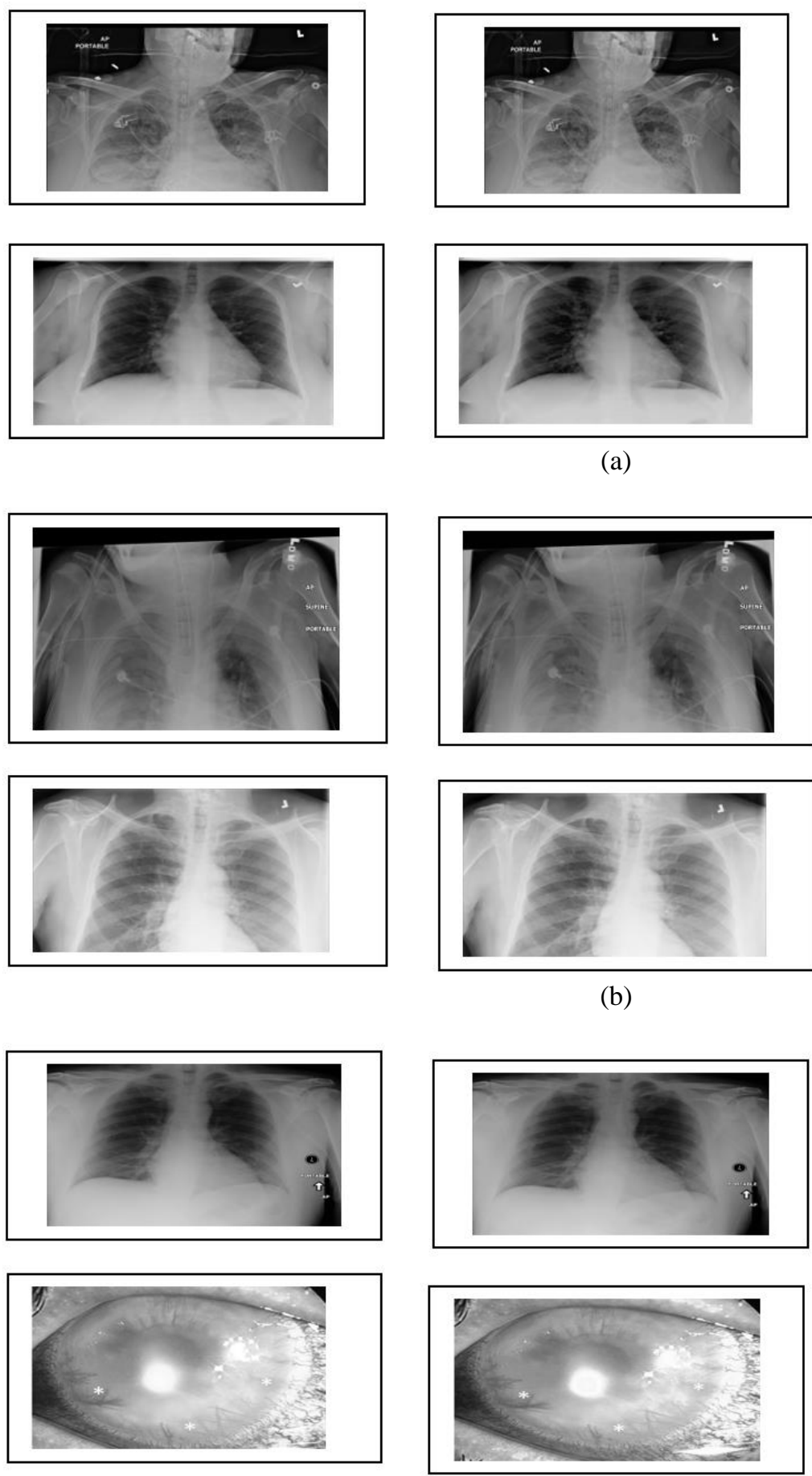

(c)

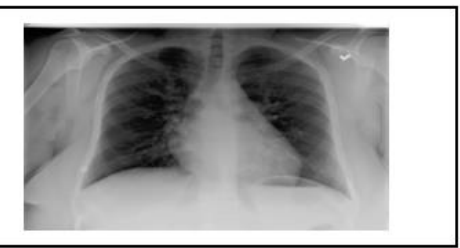

(a)
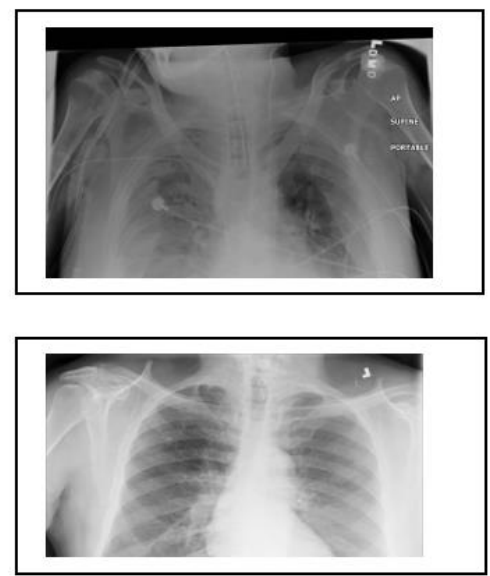

(b)

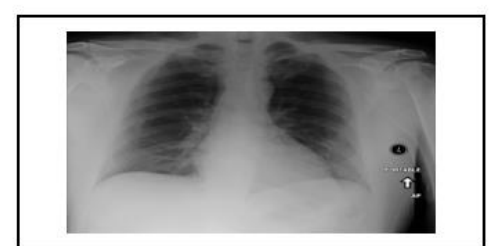

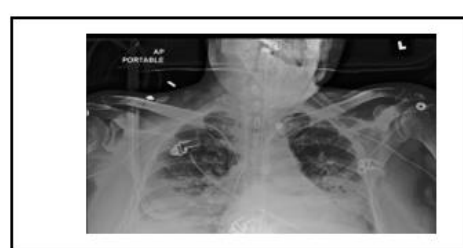
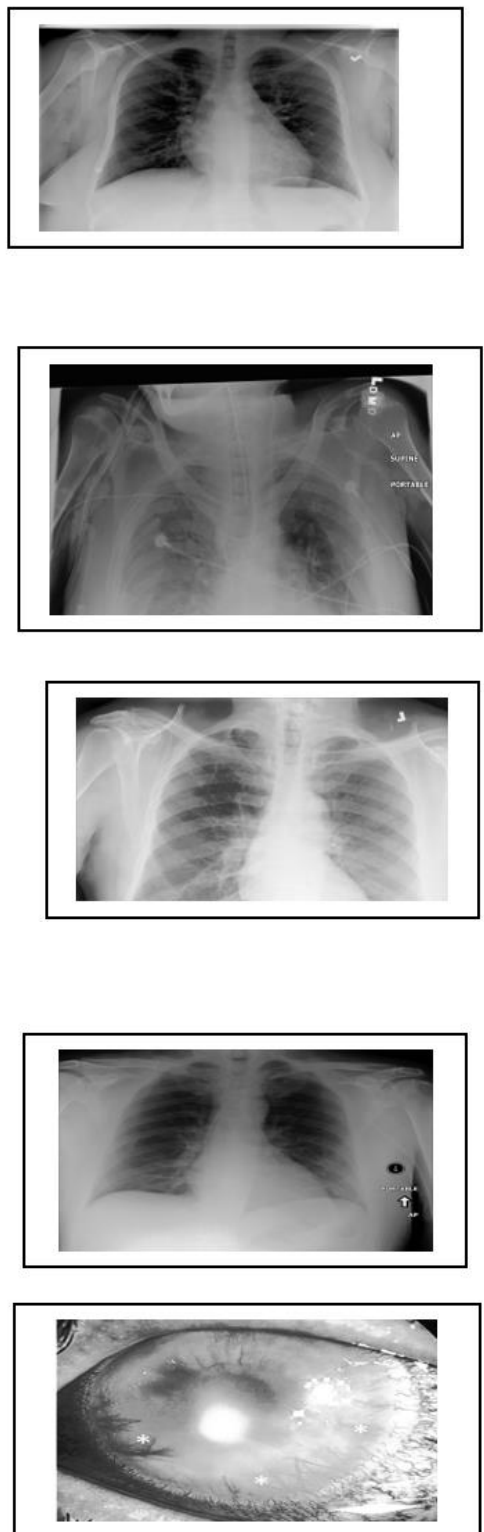

Figure 1. Simulation results; (a) original image, (b) proposed method, (c) fcnBPDFHE method

Table 1. Performance comparison between the existing and proposed method

\begin{tabular}{lccccccccc}
\hline & \multicolumn{3}{c}{ Proposed method } & \multicolumn{3}{c}{ fcnBPDFHE } & \multicolumn{3}{c}{ HE } \\
\cline { 2 - 10 } & MSE & PSNR & AIC & MSE & PSNR & AIC & MSE & PSNR & AIC \\
\hline Image (1) & 3.20 & 43.1161741 & 7.3784 & 3.68 & 31.7623903 & 7.1256 & 4.31 & 40.3241 & 7.0321 \\
Image (2) & 3.06 & 43.3091819 & 7.3470 & 8.45 & 38.8961470 & 7.0187 & 8.91 & 38.5623 & 6.6723 \\
Image (3) & 1.92 & 45.3237416 & 7.6213 & 4.34 & 41.7864858 & 7.3423 & 4.56 & 41.9834 & 6.9121 \\
Image (4) & 0.98 & 48.2522532 & 7.1253 & 10.99 & 37.7540929 & 7.2460 & 5.41 & 36.9806 & 7.1870 \\
Image (5) & 0.85 & 48.8843268 & 6.7523 & 1.56 & 46.2319488 & 6.5712 & 1.87 & 64.8912 & 6.9812 \\
Image (6) & 2.18 & 44.7748324 & 7.1134 & 4.58 & 31.2117152 & 6.9127 & 4.91 & 30.1423 & 6.9321 \\
\hline
\end{tabular}



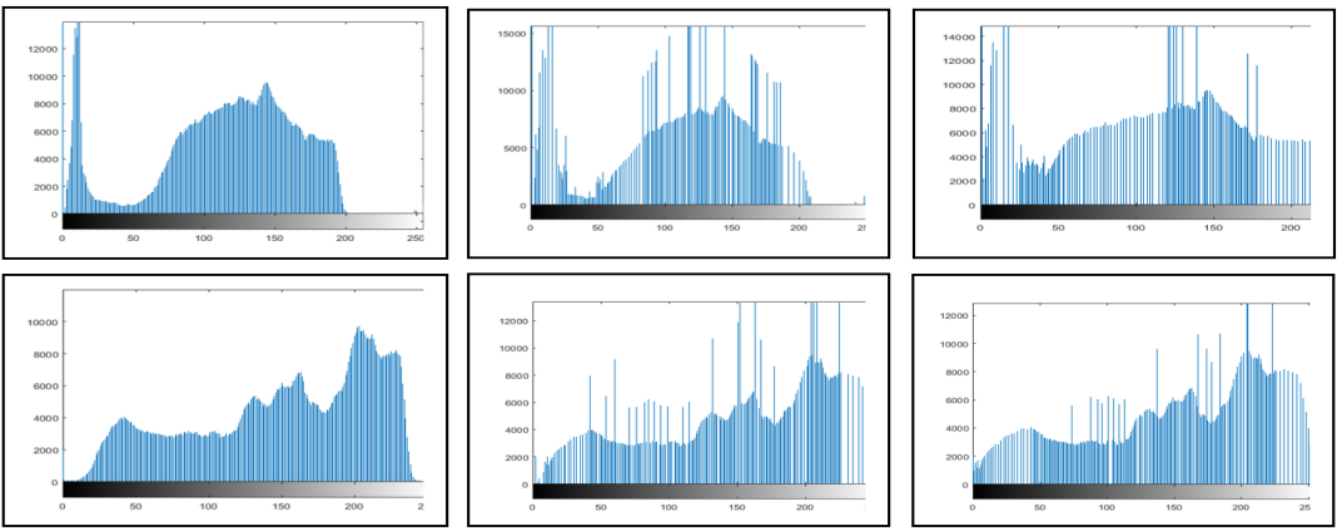

(a)
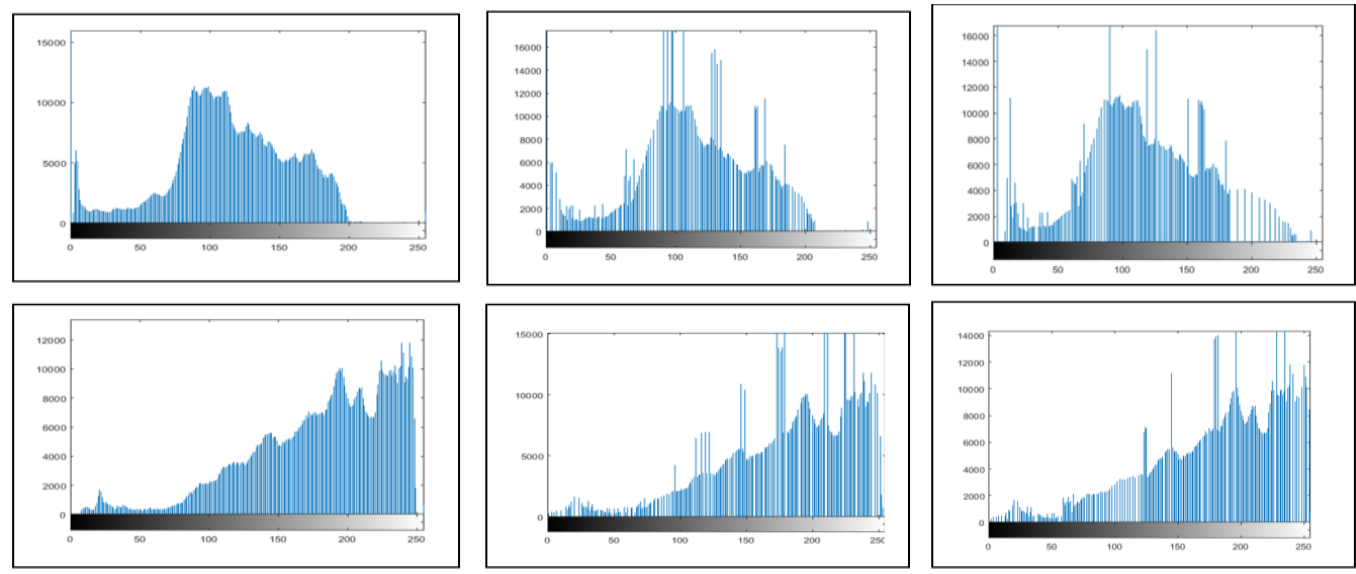

(b)
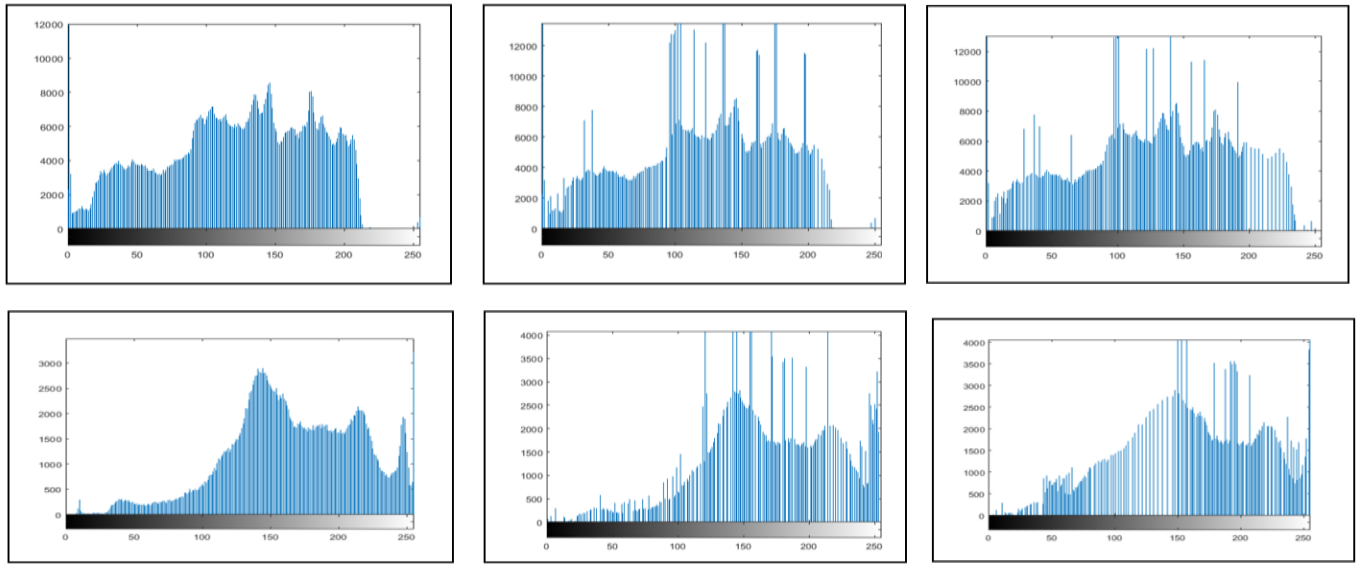

(c)

Figure 2. Simulation result of the gray scale medical images; (a) original image, (b) proposed method, (c) fcnBPDFHE method

\section{CONCLUSION}

Many image improvement strategies that can be employed for medical picture enhancement are highlighted in the proposed study. There are still many improvements and challenges required in existing techniques to get better results and improve their performance. The developed algorithm was tested on many gray medical images. These images were evaluated by the designed algorithm, and the results compared with ground truth image using many metrics such including PSNR, MSE, and AIC measures. the result of the simulation depicted that the offered algorithm gave better results than a conventional method such as (fcnBPDFHE). The proposed method can be further improved and tested on a larger dataset acquired under various conditions. 


\section{REFERENCES}

[1] U. Kumbhar, V. Patil, and S. Rudrakshi, "Enhancement of Medical Images Using Image Processing in Matlab," International Journal of Engineering Research \& Technology (IJERT), vol. 2, no. 4, pp. 2359-2364, April 2013.

[2] S. M. Hama and M. Shaban Alani "Medical Image Enhancement Based on An Efficient Approach for Adaptive Anisotropic Diffusion," International Journal of Advances in Engineering \& Technology, vol. 6, no. 3, p. 1424, July 2013.

[3] K. Sarath and S. Sreejith, "Image Enhancement Using Fuzzy Logic," IOSR Journal of Electronics and Communication Engineering (IOSR-JECE), pp. 34-44, 2017.

[4] Y. Rao, "Denoising and Enhancement of Medical Images Using Wavelets in LabVIEW," I.J. Image, Graphics and Signal Processing, vol. 7, no. 11, pp. 42-47, October 2015, doi: 10.5815/ijigsp.2015.11.06.

[5] K. Subr, A. Majumder, and S. Irani, "Greedy Algorithm for Local Contrast Enhancement of Images," in: Roli F., Vitulano S. (eds) Image Analysis and Processing-ICIAP 2005. ICIAP 2005. Lecture Notes in Computer Science, Springer, Berlin, Heidelberg, vol. 3617, pp. 171-179, 2005, doi: 10.1007/11553595_21.

[6] Tchendjou, Ghislain Takam, Rshdee Alhakim, and Emmanuel Simeu. "Fuzzy logic modeling for objective image quality assessment." In 2016 Conference on Design and Architectures for Signal and Image Processing (DASIP), IEEE, 2016, pp. 98-105.

[7] H. Darwishe, "Developing A new Methodology for optimal Facility Site Selection Analysis on Fazy Logic in GIS Environment," Al-Baath University Journal, vol. 32, no. 51, 2016.

[8] Sapna Josephus, Chelsy, and S. Remya. "Enhancement Techniques for Local Content Preservation and Contrast Improvement in Images." arXiv e-prints 2012: arXiv-1203.

[9] O. Akcay and E. O. Avsar, "The Effect of Image Enhancement Methods During Feature Detection and Matching of Thermal," The International Archives of the Photogrammetry, Remote Sensing and Spatial Information Sciences, vol. 42, p. 575, 2017, doi: 10.5194/isprs-archives-XLII-1-W1-575-2017.

[10] Abdullah-Al-Wadud, M. "A modified histogram equalization for contrast enhancement preserving the small parts in images." International Journal of Computer Science and Network Security (IJCSNS), vol. 12, no. 2, p. 1, 2012.

[11] A. Anand, "Framelet Transformed Based Satallite Image Enhancement," SSRG International Journal of Engineering and applied Scince, vol. 12, no. 6, pp. 4169-4171, 2017, doi: 10.36478/jeasci.2017.4169.4171.

[12] P. N. Badgujar and J. K. Singh, "Underwater Image Enhancement using Generalized Histogram Equalization, Discrete Wavelet Transform \& KL-Transform," International Journal of Innovative Research in Science, Engineering and Technology, vol. 6, no. 6, pp. 11834-11840, June 2017, doi: :10.15680/IJIRSET.2017.0606169.

[13] K. I. AlSaif and A. S. Abdullah, "Image Contrast Enhancement of Hsi Color Space Based on Contourlet Transform," International Journal of Information Technology and Business Management, vol. 15, no. 1, pp. 60-66, 2013.

[14] M. V. Srinu, G. N. Swetha, and M. Deepthi, "A Multi-Scale Image Enhancement Model using Human Visual System Characteristics," I.J. Image, Graphics and Signal Processing, vol. 7, no. 6, pp. 1-9, 2015, doi: 10.5815/ijigsp.2015.06.01.

[15] P. K. Shetty and V. S. V. Devi, "Image Enhancement Using MamdaniFuzzy Inference System," International Journal of Innovative Research in Computer Science \& Technology (IJIRCST), vol. 3, no. 4, pp, 37-43, July-2015.

[16] A. K. Gupta, S. S. Chauhan, and M. Shrivastava, "Low contrast image enhancement technique by using fuzzy method," International Journal of Engineering Research and General Science, vol. 4, no. 2, pp. 518-26, March 2016.

[17] K. Hasikin and N. A. M. Isa, "Enhancement of the Low Contrast Image Using Fuzzy Set Theory," 2012 UKSim 14th International Conference on Computer Modelling and Simulation, 2012, pp. 371-376, doi: 10.1109/UKSim.2012.60.

[18] S. Raj, S. Raj, and S. Kumar, "An Improved Histogram Equalization Technique for Image Contrast Enhancement, International Conference on Communication and Computing, 2015.

[19] Salem, Nema, Hebatullah Malik, and Asmaa Shams. "Medical image enhancement based on histogram algorithms." Procedia Computer Science, vol. 163, pp. 300-311, 2019.

[20] D. Ngo, S. Lee, and B. Kang, "Light Stretch Algorithm for Image Quality Enhancement," Proceedings of the 4th International Conference on Virtual Reality, pp. 56-60, February 2018, doi: 10.1145/3198910.3198921.

[21] N. V. Lopes, P. A. M. do Couto, H. Bustince, and P. Melo-Pinto, "Automatic Histogram Threshold Using Fuzzy Measures," in IEEE Transactions on Image Processing, vol. 19, no. 1, pp. 199-204, Jan. 2010, doi: 10.1109/TIP.2009.2032349.

[22] H. Yoon, Y. Han, and H. Hahn, "Image contrast enhancement based sub-histogram equalization technique without over-equalization noise," World Academy of Science, Engineering and Technology, vol. 50, pp. 176-182, 2009.

[23] B. Oktavianto and T. W. Purboyo, "A study of histogram equalization techniques for image enhancement," International Journal of Applied Engineering Research, vol. 13, no. 2, pp. 1165-1170, 2018.

[24] S. Souverville, J. A. Rosales, F. J. Gallegos, M. Dehasa, I. V. Hernández, and L. V. Lozano, "Fuzzy Logic Applied to Improvement of Image Resolution using Gaussian Membership Functions," Res. Comput. Sci., vol. 102, pp. 7788,2015

[25] A. H. Thary, "Satellite Image Classification Using K-Means and SVD Techniques," A Thesis, 2014.

[26] J. Zhang and Z. Ma, "Hybrid Fuzzy Clustering Method Based on FCM and Enhanced Logarithmical PSO (ELPSO)," Computational intelligence and neuroscience, vol. 2020, 2020, doi: 10.1155/2020/1386839.

[27] Zhang, Jian, and Zongheng Ma. "Hybrid Fuzzy Clustering Method Based on FCM and Enhanced Logarithmical PSO (ELPSO)," Computational intelligence and neuroscience, 2020. 